\title{
Dermoscopic findings in a case of multiple subungual fibromas
}

\author{
Muazzez Çiğdem Oba ${ }^{\circledR}$, Tuğba Kevser Uzunçakmak', Mehmet Sar², Server Serdaroğlu ${ }^{1}$ \\ ${ }^{1}$ Department of Dermatology and Venereology, Cerrahpaşa Medical Faculty, Istanbul University-Cerrahpaşa, Istanbul, Turkey. ${ }^{2}$ Department of \\ Pathology, Cerrahpaşa Medical Faculty, Istanbul University-Cerrahpaşa, Istanbul, Turkey.
}

\begin{abstract}
Periungual and subungual fibromas, also known as Koenen tumors, are diagnostic findings of tuberous sclerosis. The clinical appearance and histological features that characterize ungual fibromas are well defined. However, dermoscopic findings of these benign tumors have not been reported previously. Here we report a rare presentation of multiple subungual fibromas of all fingers in a developmentally delayed patient with tuberous sclerosis along with the dermoscopic features of the ungual tumors.
\end{abstract}

Keywords: dermatopathology, dermoscopy, nail disease, nail genetic disorder, nail tumor, tuberous sclerosis

Received: 15 August 2020 | Returned for modification: 4 October 2020 | Accepted: 13 October 2020

\section{Introduction}

Periungual and subungual fibromas, also known as Koenen tumors, are diagnostic findings of tuberous sclerosis (TS), an autosomal dominantly inherited disease involving multiple organs including the brain, eyes, heart, lungs, kidneys, skin, and skin appendages (1). The classic triad of TS consisting of seizures, developmental delay, and angiofibromas occurs in only $29 \%$ of patients (2). Thus, in mild or incomplete forms of the disease, skin findings are of utmost importance when suspecting a diagnosis of TS (3). Hypomelanotic macules, angiofibromas, ungual fibromas, and shagreen patches are major cutaneous signs of TS (4). The clinical appearance and histological features that characterize ungual fibromas are well defined $(1,5)$. However, dermoscopic findings of these benign tumors have not been reported previously.

\section{Case report}

A 28-year-old man with known TS and developmental delay presented to the dermatology clinic with subungual tumors and nail changes on all fingernails. The subungual tumors had been present for 5 years, progressively growing in size and number. Upon clinical examination, all his fingers had multiple firm skin-colored subungual papules, ranging between 1 and $4 \mathrm{~mm}$ in size (Figs. 1a, 1b). The fingernail plates showed yellowish discoloration and thickening, and they were lifted up due to extensive bulky lesions. In contrast, the ungual tumors involving his toes were few in number and periungually located (Fig. 1c). Subungual hyperkeratosis and yellow discoloration were noted on the large toenail along with thinning of the nail plate and longitudinal ridging of other toenails. Dermoscopic pictures were taken using a DermLite DL4 dermatoscope with $\times 10$ magnification (3Gen LLC, Dana Point, CA, USA) (polarizing mode). Dermoscopy of the fingernails revealed pink homogenous areas comprising polymorphous vessels (dotted, curved, and glomerular vessels) surrounded by a scaly whitish-yellow collarette and hemorrhage. Some of the papules showed yellow homogenous areas at their apical part (Figs. 1d and 1e). Dermoscopy of the toenails revealed pink homogenous areas with glomerular vessels and a scaly collarette (Figs. 1f, 1g). A punch biopsy specimen obtained from the third fingernail bed revealed marked epidermal acanthosis and hyperkeratosis along with extensive fibrosis and ectatic vessels in the dermis. Stellate fibroblasts were present between the dense collagen fibers, which had a vertical orientation (Fig. 2). The histopathological findings were consistent with the diagnosis of Koenen tumor. The family was reassured that the condition was associated with TS. They did not request medical treatment.

\section{Discussion}

Koenen tumors are benign fibromas of the distal digits, occurring in approximately half of patients with TS (1). However, these tumors may also occur independently of TS. They often appear after puberty as red to skin-colored firm nodules around or under the toenails, and less commonly the fingernails. They are generally 5 to $10 \mathrm{~mm}$ in length, but they may be very large (6). Periungual fibromas are more common than subungual fibromas. Other nail findings of TS include longitudinal grooves with or without periungual fibromas, red comets, longitudinal leukonychia, and splinter hemorrhages (7).

Trauma is thought to play a substantial role in the formation of ungual fibromas. Periungual fibromas occur most commonly on the fifth toe, and subungual fibromas on the thumb. This non-random distribution of the lesions is attributed to the occurrence of ungual tumors on trauma-prone sites. In addition, some patients report a history of crushing trauma preceding the development of fibromas (7). Our patient was picking and pressing the tumors using foreign materials such as wire. We believe this self-mutilating behavior may have triggered the extensive fibromas.

Histopathologically, ungual fibromas are covered with an acanthotic and hyperkeratotic epidermis. A stroma containing capillaries surrounded by loose collagen fibers distally and dense close-packed collagen fibers proximally is characteristic of Koenen tumors (5). It is controversial whether Koenen tumors are a type of fibrokeratoma (8).

In our patient, dermoscopy revealed pale pink homogenous areas surrounded by a hyperkeratotic whitish-yellow collarette. Glomerular, curved linear and dotted vessels were also observed in the pink homogenous areas (Figs. 1d-1g). The apical part of some tumors showed a yellow homogenous area, corresponding to epidermal hyperkeratosis. We considered most dermoscopic findings to be similar to those of acquired digital fibrokeratoma $(9,10)$. 
Clinically, the differential diagnosis of Koenen tumors most commonly includes viral warts. The lack of thrombosed capillaries and the fusiform shape of Koenen tumors with rounded and tapering ends help in their clinical differentiation. Dermatofibroma, superficial acral fibromyxoma, and subungual neurofibroma are rarely encountered clinical differential diagnoses of Koenen tumors, and histopathological examination is needed to establish the diagnosis (8).

Koenen tumors may cause functional and cosmetic problems. Surgical excision, electrodessication, shave and phenolization, ab- lative lasers ( $\mathrm{CO}_{2}$ laser), and topical mTOR (sirolimus) inhibitors are the treatment options (4). However, lesions tend to recur (2).

In conclusion, our patient had a large number of subungual fibromas of the fingernails. This was a relatively rare presentation of Koenen tumors, which are mostly periungual and involve the toes. Unusually large or extensive ungual tumors are mostly seen in TS patients with developmental delays (6). Although not reported previously, dermoscopic findings can help in the diagnosis of ungual fibromas.

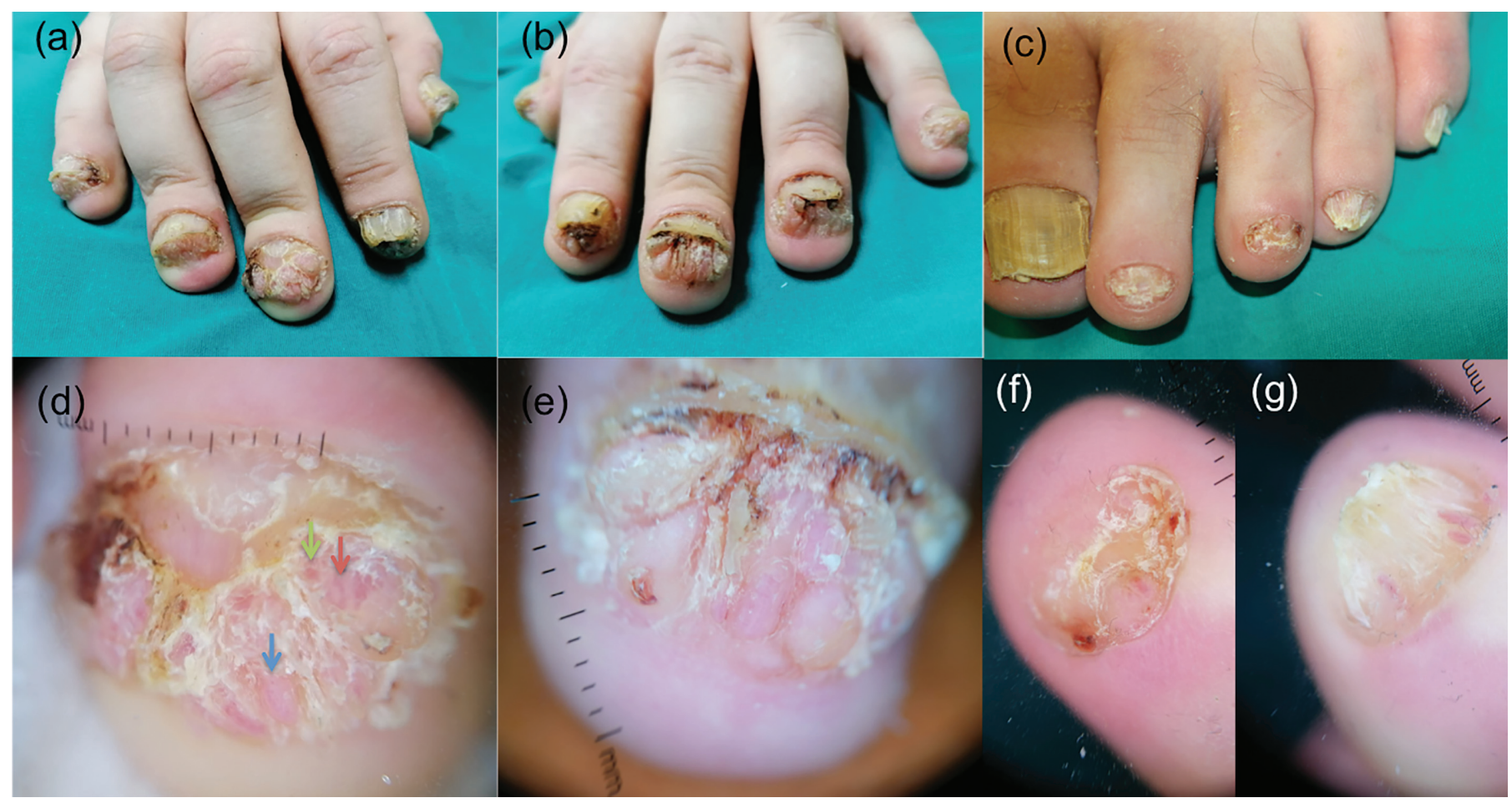

Figure 1 | Multiple bilateral subungual fibromas on the right and left hands (a, b); several periungual fibromas on the left foot (c); pink homogenous areas with polymorphous vessels on the fingers (green arrow: glomerular vessels, red arrow: curved linear vessels, blue arrow: dotted vessels), scaly collarette, and traumatic hemorrhage (d, e); pink homogenous areas with glomerular vessels and scaly collarette on the toenails (f, g).

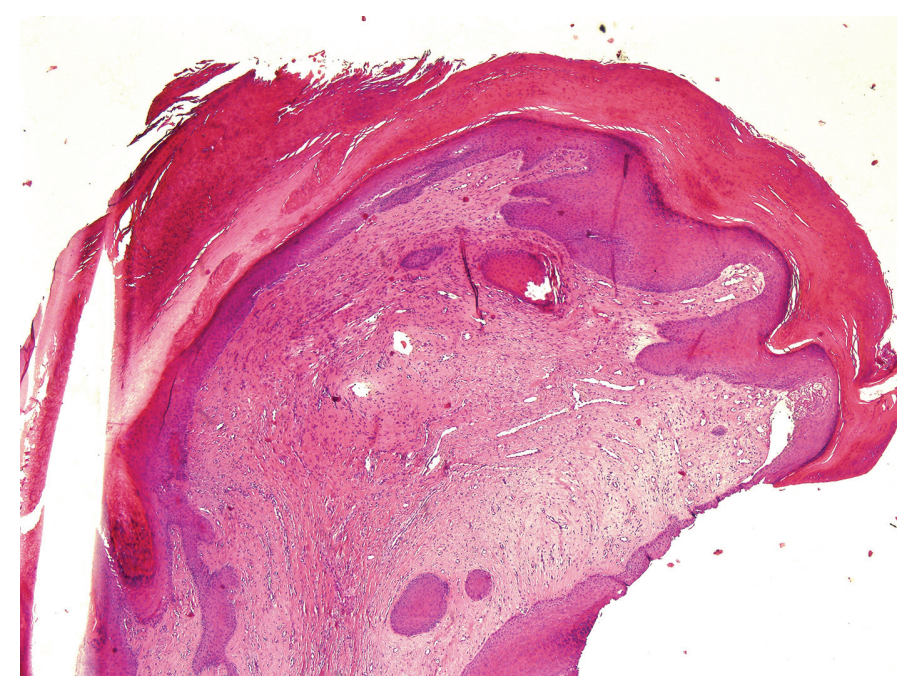

Figure 2 | Epidermal acanthosis and hyperkeratosis with marked fibrosis and ectatic vessels in the dermis (H\&E, $5 \times$ ).

\section{References}

1. Baran R, Richert B. Common nail tumors. Dermatol Clin. 2006;24:297-311.

2. Hake S. Cutaneous manifestations of tuberous sclerosis. Ochsner J. 2010;10: 200-4.
3. Quist SR, Franke I, Sutter C, Bartram CR, Gollnick HP, Leverkus M. Periungual fibroma (Koenen tumors) as isolated sign of tuberous sclerosis complex with tuberous sclerosis complex 1 germline mutation. J Am Acad Dermatol. 2010;62:159-61. 
4. Cardis MA, Deklotz CMC. Cutaneous manifestations of tuberous sclerosis complex and the paediatrician's role. Arch Dis Child. 2017;102:858-63.

5. Kint A, Baran R. Histopathologic study of Koenen tumors: are they different from acquired digital fibrokeratoma? J Am Acad Dermatol. 1988;18:369-72.

6. Aste N, Pau M, Aste N, Biggio P. Tuberous sclerosis with unusual giant ungual fibromas. J Eur Acad Dermatology Venereol. 2003;17:100-1.

7. Aldrich CSL, Hong CH, Groves L, Olsen C, Moss J, Darling TN. Acral lesions in tuberous sclerosis complex: Insights into pathogenesis. J Am Acad Dermatol. 2010;63:244-51.
8. Shih S, Khachemoune A. Acquired digital fibrokeratoma: review of its clinical and dermoscopic features and differential diagnosis. Int J Dermatol. 2019;58:151-8.

9. Rubegni P, Poggiali S, Lamberti A, Chiantini A, De Paola M, Peccianti C, et al. Dermoscopy of acquired digital fibrokeratoma. Australas J Dermatol. 2012;53:47-8.

10. Hayashi K, Matori S, Kariya Y, Sonosaki T, Yamaguchi S, Hagiwara K, et al. Dermoscopic observation of acquired digital fibrokeratoma developed on the dorsum of the fourth left toe. J Dermatol. 2016;43:107-8. 